\title{
Muscle Electro Stimulator for the Reduction of Stretch Marks
}

\author{
Paima-Sahuma Jaime ${ }^{1}$, Duran-Berrio Linder ${ }^{2}$, Palacios-Cusiyunca Chrisstopher ${ }^{3}$, Roman-Gonzalez Avid ${ }^{4}$ \\ Electronics and Telecommunications Department, Universidad Nacional Tecnológica de Lima Sur, Lima, Peru ${ }^{1,2,3}$ \\ Aerospace Sciences and Health Research Laboratory (INCA-Lab), Universidad Nacional Tecnológica de Lima Sur, Lima, Peru ${ }^{4}$
}

\begin{abstract}
The problem of stretch marks is generated because the skin stretches abruptly in a short time; this change causes the skin to deform and widen, forming a roughness. This roughness is what is known as stretch marks. This document arose from the need to reduce the deformation in the skin that many people suffer. This deformation mainly is due to overweight, pregnancy, or during adolescence due to rapid growth. In this document, a device will be designed that will have the task of reducing skin roughness. One will use electro-stimulation as the primary technique to apply electrical impulses. This device can limit and control the electrical signals produced to control the movement of muscle fibers and skin. The results obtained show a remarkable reduction of stretch marks in one people after the application of electrical stimuli with the device. Research shows promising results.
\end{abstract}

\section{Keywords-Electro stimulator; stretch marks; muscle fibers}

\section{INTRODUCTION}

From a physiological point of view, stretch marks are caused by stretching of the middle layers of skin, internal skin due to pregnancy, puberty, rapid growth, and sudden weight gain or weight training.

Stretch marks are skin atrophies, very visible thinning of the skin. These marks occur as a result of breakage and partial loss of collagen fibers and elastin in the affected region. This situation resulting in decreased skin cohesion and yielding said area tension forces of muscle mass that support the skin [1].

Stretch marks are found mainly in the abdomen, legs, and breasts. In reality, they are a pathological state of the connective tissue of the dermis, isolated by excessive localized fibrosis in the form of ropes in response to breakage and poor fiber quality. It can be said that they are scars of this tissue. If stretch marks, when formed, are violet or purple, it indicates that the dermis still has a blood supply. If then they are roses, it also has irrigation.

When there is a breakdown of collagen and elastin fibers, the skin weakens and becomes susceptible to permanent scars, as an old rubber band tends to lose its elasticity.

The devices commonly used to treat stretch marks problems have recently gained popularity in the consumer market. But they are complex to use and have a high cost. Some are invasive and, in turn, are accompanied by a longterm treatment that the patient can't pay.

In this article, a device that uses electro stimulation as the primary technique to reduce stretch marks will be shown. In
Section II, one will focus on explaining the design of the electro stimulator. The electroestimulator is composed of electronic devices at low cost, the frequency ranges that are of reference to know what parameters to use and get the desired result will be defined. Different types explained waves and their effects on muscle and nerve. Section III will debate on the use of electro stimulator and treatment time. In Section IV, the results obtained with the electro stimulator efficiency and to reduce streaks in the different parts of the body where they are located will be explained. The conclusions derived through research to finalize detailed in Section $\mathrm{V}$, and also mentioned recommendations to consider and take into account.

\section{Methodology}

The flow chart used for the development of the project is shown in Fig. 1. The flow chart begins with a processing and control stage that concludes in a test stage.

Concerning the processing and control of the signals, the placement of electrodes in the area of the body that has stretch marks is performed. Then the electrical signals produce the vibration of the electrodes, and finally, the signals are regulated by using the frequency table of impulses (Table I). The tests were performed on patients affected with stretch marks, obtaining favorable results.

\section{A. Circuit Design}

Muscular electrostimulation is a technique that involves the application of electrical pulses by using controlled electrical current to the induction of muscle contraction [2]. The pulses are generated on a device that applies electrodes on the skin near to muscles that are intended to stimulate. They mimic the action impulses from the central nervous system, causing muscle contraction.

The electrodes generally adhere to the skin. EEM is a form of electrotherapy or muscle training [3]. Several authors cite it as a complementary technique for the treatment of muscle; there are numerous studies published on the matter. It was found that this technique could be used as therapy for people with particular rehabilitation-related problems or muscle tissue or muscle problems such as stretch marks disorders. Currently, muscle electro stimulation used for recovery purposes centers or as a form of muscle training.

This circuit design seeks to generate high voltage pulse voltage and low current that is not detrimental to the sensitivity of the human body so that the muscles can contract. The circuit works with $220 \mathrm{v}$ in the primary of the transformer, and this so that the output of the transformer we reduced 6.9 or 12 -volt 
alternating voltage, the diodes are responsible for rectifying and smoothing the electrolyte capacitors. The primary function of the circuit of "Fig. 2" is to generate pulses of short duration that are perceived through the electrodes, which, when connected to the body, can contract the muscle.

We design a circuit with the following components:

- 1 LM556

- 1 audio transformer $110 \mathrm{v} / 12 \mathrm{v}$ to 100 milliamps (or less better between 60 to 100miliamperios)

- Resistors 1k ohm, 1k ohm, 390 ohms, 330 ohms, 33k, $220 \mathrm{hm}$ (3), 50k

- Capacitors 100 Nano farad, 10 microfarads

- 3 led

- $5 \mathrm{k}$ potentiometers, $1 \mathrm{M}, 10 \mathrm{k}$

- Transistors 2N2222, 2N3053

- Two 1N4004 diodes

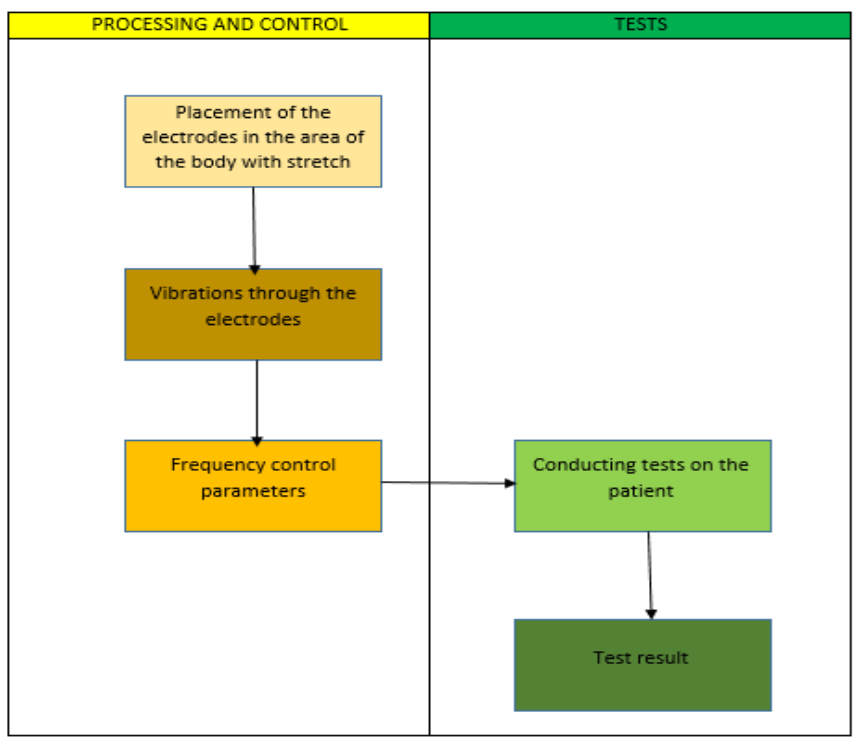

Fig. 1. Diagram Flow.

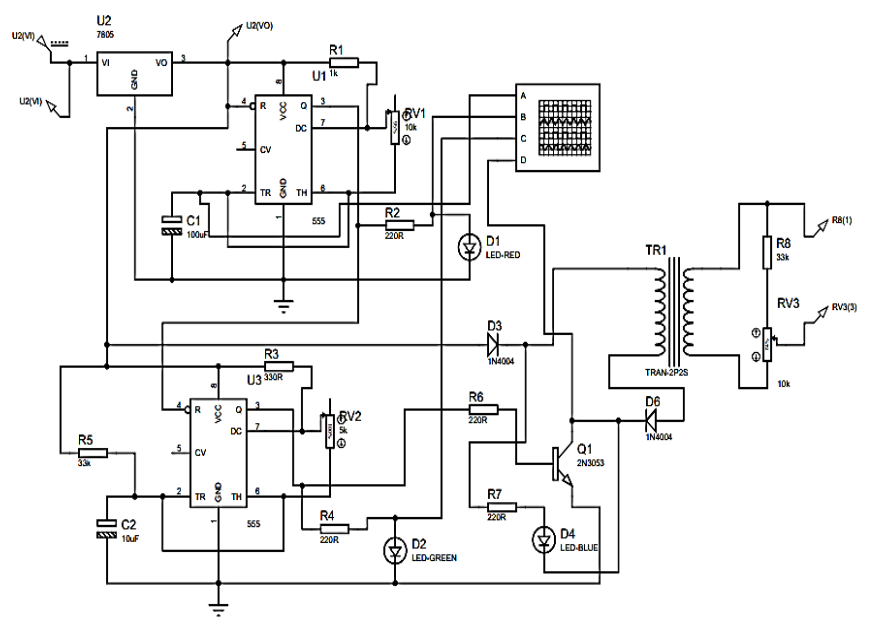

Fig. 2. Electro Stimulator Circuit.
In "Fig. 3", we design the circuit using the Proteus Design Suite, software of electronic design automation, and this to create the tracks on the circuit.

In "Fig. 4", we can see a final prototype 3D, how will be the circuit once it is implemented with electronic components.

\section{B. Operating Principle}

The critical aspect of electro stimulation lies in knowing in detail the program that you want to work because its effects can be varied [4]. In this sense, if you're looking to lose weight, you should know that the program to follow will be the type of stretch marks, as is the one who can help in this task. This specific program improves the ability to metabolize fats in your body and pass through the anterior muscle to the patient's skin. Also, other sections that one should not overlook, when resorting to the sessions with devices electro stimulation, is that usually, this method is only able to stimulate a muscle group at a time. This situation implies a significant dedication and time consuming to exercise full musculature.

In "Fig. 5", we have an operation diagram which indicates the work to conduct the electro once the electrodes are connected to the body area affected by stretch marks.

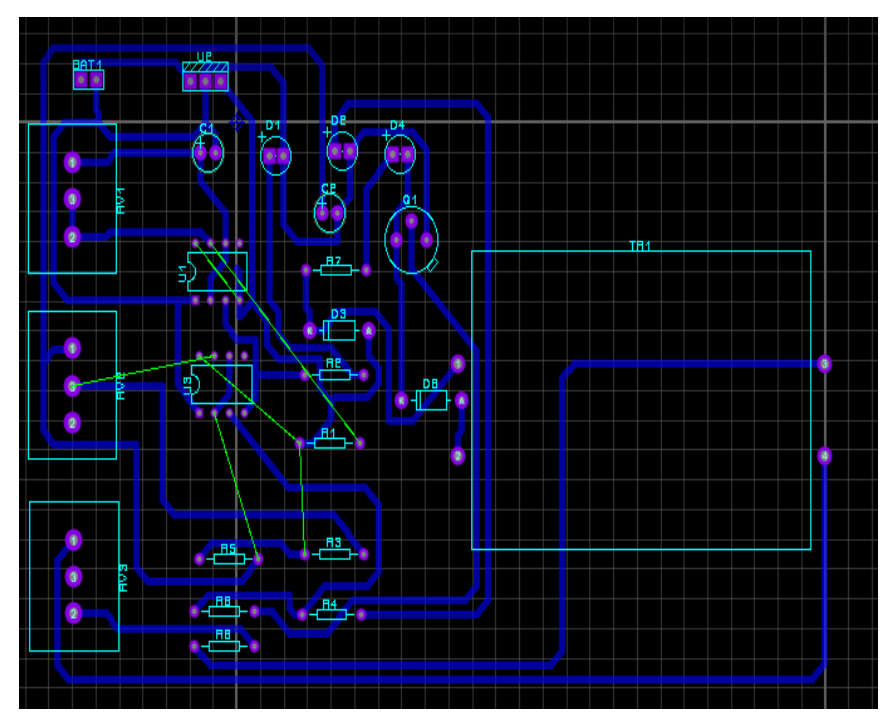

Fig. 3. Track Circuit Design.

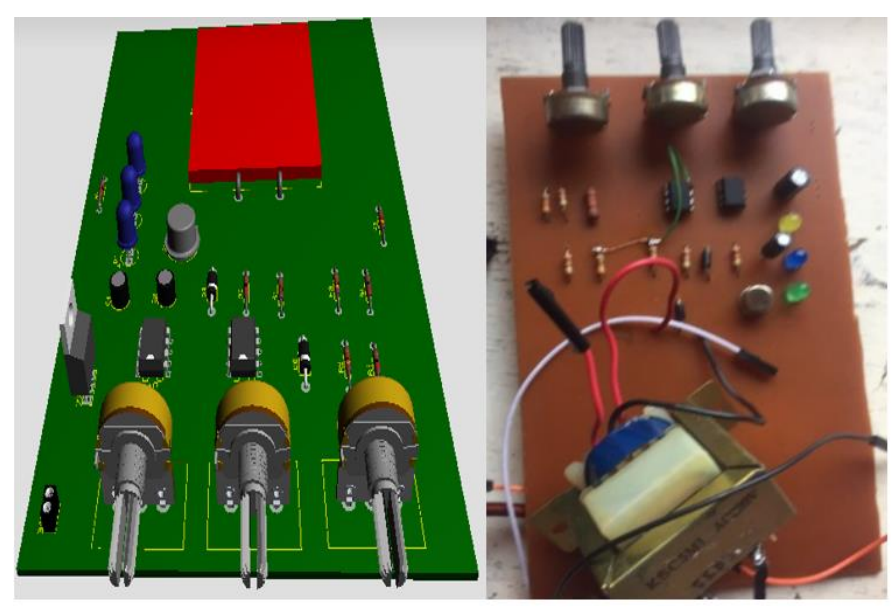

Fig. 4. 3D Simulation Circuit and Implementation of Circuit. 


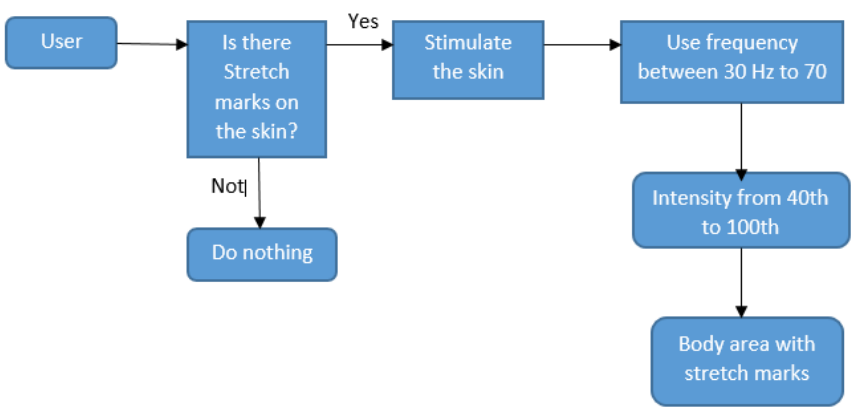

Fig. 5. Operating Diagram.

\section{Electro Stimulation Signal}

The signal to produce muscle stimulation would be achieved with electric waves, and thus a reaction in the affected area would be performed. One must apply the electrical parameters that will be used. It must be minimum energy, intensity, and duration, and one must locate the characteristics of the optimal flows and comply with its fundamental law. The most advanced electro stimulator has already programmed the $\mathrm{Hz}$, depending on the frequency. It has the appropriate terminology to improve sports performance (explosive strength, strength, strength resistance, hypertrophy). The search for an aesthetic improvement (muscle firmness, toning, and bodybuilding) on functional recovery and improvement of the quality of life (back pain, neck pain, active recovery, relaxation, drainage) [5]. The electro stimulator of the device recreates the signals that are sent through electrodes in pulses and in response. The muscle reacts with a contraction. The tissue cannot distinguish if the command comes from outside the brain.

\section{Pulse Frequency}

Is the number of pulses which are repeated at a given time is measured in Hertz? In the table, we set the frequency range that the stimulator should work. While the frequency is higher, the greater the strength and power. Typically, one speaks of three types of fiber types working in the following frequencies:

a) Slow fibers: His frequency begins from the $10 \mathrm{~Hz}$, and peaks at $33 \mathrm{~Hz}$ should be clear that always stimulates both slow fibers.

b) Mixed fibers: His tetanization starts at $20 \mathrm{~Hz}$ and ending at $50 \mathrm{~Hz}$, frequencies used to improve strength.

c) Fast fibers: Their frequency starts at $33 \mathrm{~Hz}$ and ends at $66 \mathrm{~Hz}$ However, these are given for a sedentary person values; Sportsmen higher frequencies are used.

\section{E. Electrodes EMS}

The electrodes are generally metal plates used as a driver responsible for making contact with a circuit sector nonmetallic type, but electrolyte, as the human body (Table I). EMS means electrical muscle stimulation is a technique that promotes muscle contractions by applying electrical impulses. EMS is commonly used for medical and physiotherapy and sport complement treatments; therefore, therapeutic purposes, sports or aesthetic, anti-cellulite, and drainage [6].
TABLE. I. IMPULSE FREQUENCY RANGE

\begin{tabular}{|l|l|}
\hline Frequency $(\mathbf{H z})$ & Effects of frequencies according to the range used \\
\hline 1 to 10 & $\begin{array}{l}\text { Relaxation increased blood flow and segregation of } \\
\text { endorphins. }\end{array}$ \\
\hline 10 to 20 & $\begin{array}{l}\text { Improving aerobic endurance muscle (muscle oxidative } \\
\text { capacity). }\end{array}$ \\
\hline 20 to 50 & $\begin{array}{l}\text { Improved muscle tone, muscle definition and muscle } \\
\text { firmness (aesthetic effects and early stages of } \\
\text { rehabilitation). }\end{array}$ \\
\hline 40 to 70 & $\begin{array}{l}\text { Lactic improved capabilities muscle and increase muscle } \\
\text { volume. }\end{array}$ \\
\hline 70 to 120 & Improving maximum strength. \\
\hline 90 to 150 & Improve explosive, reactive elastic force. \\
\hline
\end{tabular}

In the body, currents are produced by the movement of ions. The flow of electrons creates a wire for operating a computer.

In "Fig. 6", we show the kind of electrodes we use to the electro stimulator, which are suction electrodes that are useful to lower resistance and increase the electrical conductivity of the skin.

\section{F. Feeding Step}

A source that allows the correct operation between blocks mentioned above will be developed. Voltages to be used will be of $9 \mathrm{seg}$ and 5 volt DC. I know it will require a linear regulator that will allow me to get the 5v. DC. The transistor is using the $2 \mathrm{~N} 3053$ voltage converter.

\section{G. Waveforms}

Currently, the stimulator can work with different types of waves are grouped as follows:

a) Galvanic wave: It is the first form of electric current. It uses low frequency with a particular characteristic that is to maintain a constant voltage at a particular time, as it is observed in "Fig. 7".

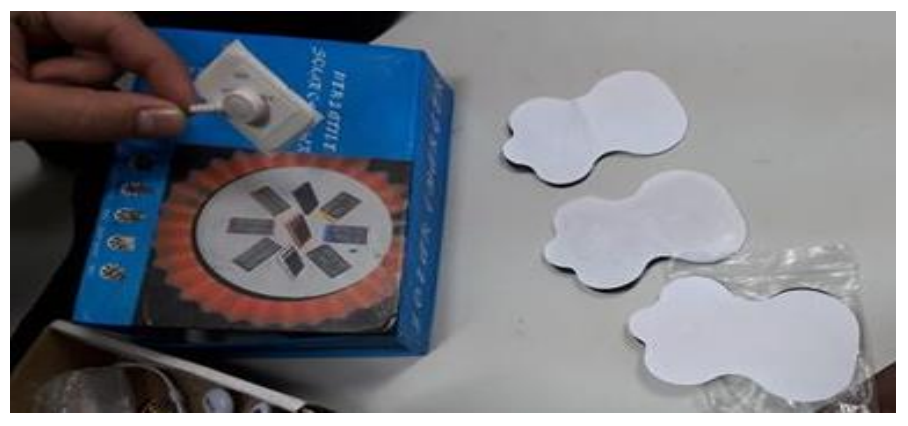

Fig. 6. Suction Cup Electrodes.

b) Faradic wave: The wave is asymmetric, low voltage and works in frequency approximating $50 \mathrm{~Hz}$, which is shown in "Fig 8". It has a direct application to the muscle to contract it so well innervated.

c) Interferential waves: Two alternating signals having medium frequency shown in "Fig. 9", varies with slight differences. When the two signals cross a modulated 
therapeutic current is produced at low frequency, providing a fixed frequency signal with a variable.

d) Monopolar square interrupted galvanic wave: The monophasic waveform is a square pulse shown in "Fig. 10".

e) Monopolar triangular interrupted galvanic wave: It is a monophasic continuous wave which is shown in Fig. 11. It has a succession of unmodulated pulses and which has a low frequency.

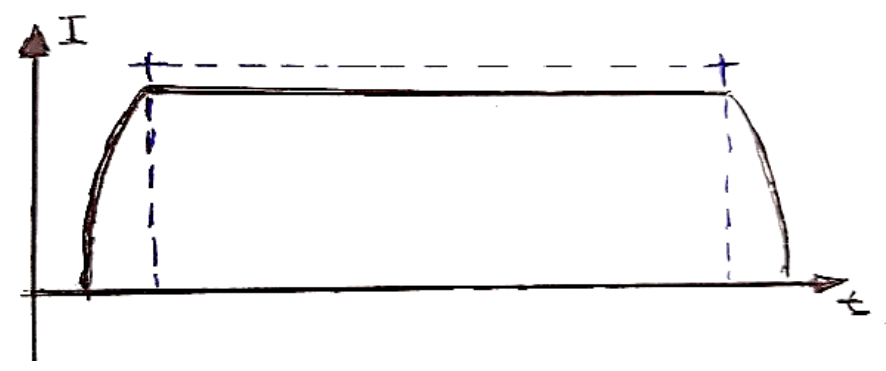

Fig. 7. Galvanic Current.

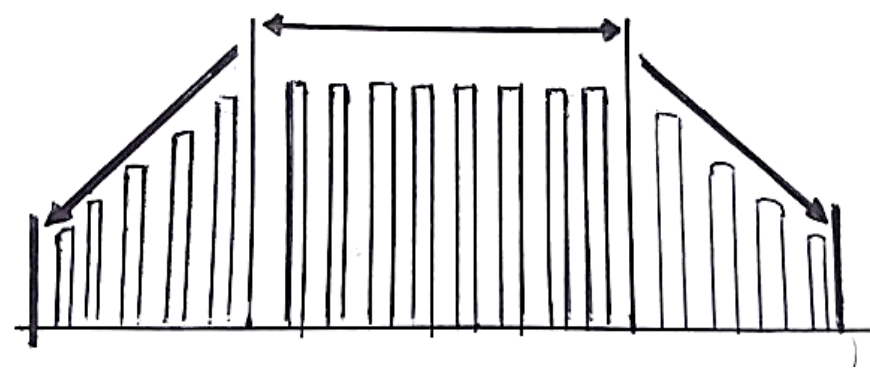

Fig. 8. Faradic Wave Train.

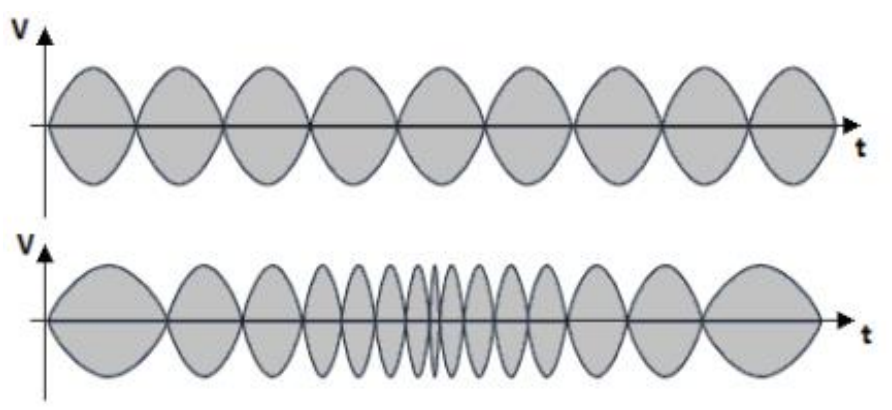

Fig. 9. Interferential Wave Modulation [7].

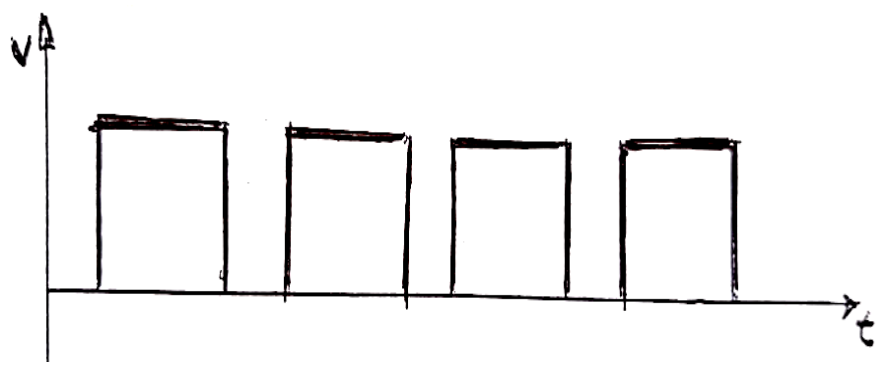

Fig. 10. Monophasic Square Wave.

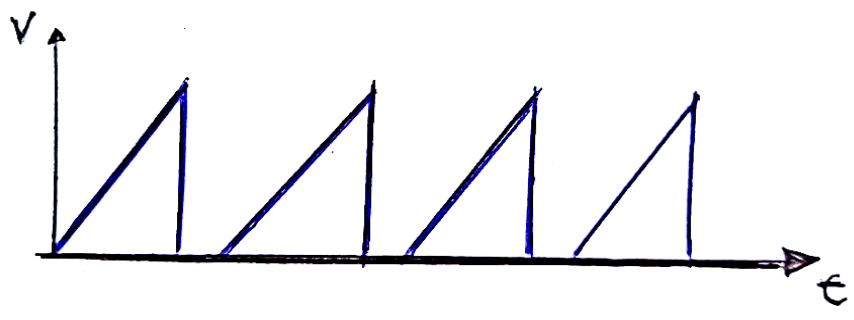

Fig. 11. Triangular Wave Monophasic.

\section{RESULTS}

Implantation of electrodes in the subject test or patients with this disease of the skin as testing time was that the use was enacted. The idea of reducing or appease the deformation of the skin (stretch marks or whitish lines). That takes place in the process of the same system to the muscles that you get to visualize the skin. One sees that as the results look will be established just said and studied since the beginning of this paper.

The classic question of whether the circuit worked or not, with the time developed a well-implemented circuit that was tested in physics. From there, the research was started to make the paper established, with the time we saw that if This use could reduce the level of stretch marks.

When contracting the muscle makes these same looks oblige to move forcefully. So that they can pass blood, it needs the skin and can see that is bloodying. One needs to see those lines with blood become clogged, and thus the muscle meets the function no longer form whitish lines on the skin, and no more streaks are seen in a long time.

It was tested as the time use also affects the nervous state and can produce Parkinson's too involuntary movement. To avoid this kind of damage long-term type of procedure, which are sessions established by professionals of this branch are available and see how the patient's body behaves.

It will be seen as the time that this will help not only on the part of the biomedical but on the physical side. The blow to the muscle also helps the exercises that can be used in the gym, as this could give movements muscles could see it improve over time.

When the stimulation session is done, it should stimulate isometrically to thereby the muscle is fixed and can prevent the movement that causes contraction.

The electro stimulator that was designed has high levels of muscle contraction, which is suitable for therapies seen.

Table II shows a picture, which will indicate how often you work, time, and the number of sessions is estimated to reduce the use of electro stretch marks shown.

In "Fig. 12", the part of the Axillary nerve shown with problems of stretch marks. To reduce the stretch marks was treated with the electro stimulator, and thus definite reduction of stretch marks was in the "Fig. 13".

In "Fig. 14", connected to the outputs of the electro stimulator, with two oscilloscope channels were shown on channel 1 (yellow signal). Square interrupted galvanic waves 
of "Fig. 10", and in channel 2 (blue signal) galvanic waves interrupted triangular of "Fig. 11".

TABLE. II. RAME OF REFERENCE FOR ELECTRO STIMULATOR USE IN AREAS OF THE BODY AFFECTED BY STRETCH MARKS

\begin{tabular}{|l|l|l|l|}
\hline $\begin{array}{l}\text { Part of the body } \\
\text { affected }\end{array}$ & $\begin{array}{l}\text { Frequency } \\
(\mathbf{H z})\end{array}$ & $\begin{array}{l}\text { Electro stimulator } \\
\text { usage time } \\
\text { (minutes) }\end{array}$ & $\begin{array}{l}\text { Number of } \\
\text { sessions }\end{array}$ \\
\hline Axillary nerve & $40-50$ & 10 & 15 \\
\hline Muscle epitrochlear & 50 & 10 & 10 \\
\hline Rectus & 50 & 15 & 15 \\
\hline Oblique muscle & 50 & 10 & 15 \\
\hline Buttocks & $50-60$ & 15 & 15 \\
\hline Gracilis muscle & $50-80$ & 15 & 15 \\
\hline Vastus muscle & $20-50$ & 15 & 20 \\
\hline Tensor fascia & 50 & 10 & 15 \\
\hline
\end{tabular}

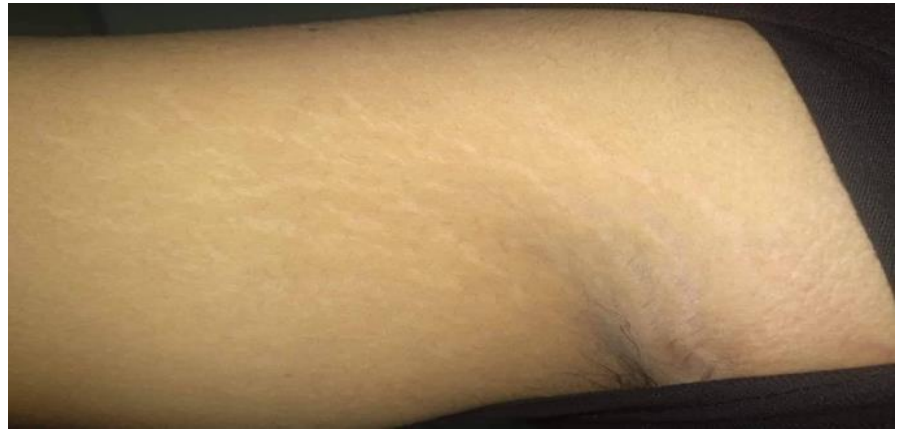

Fig. 12. Stretch Mark in Axillary Nerve.

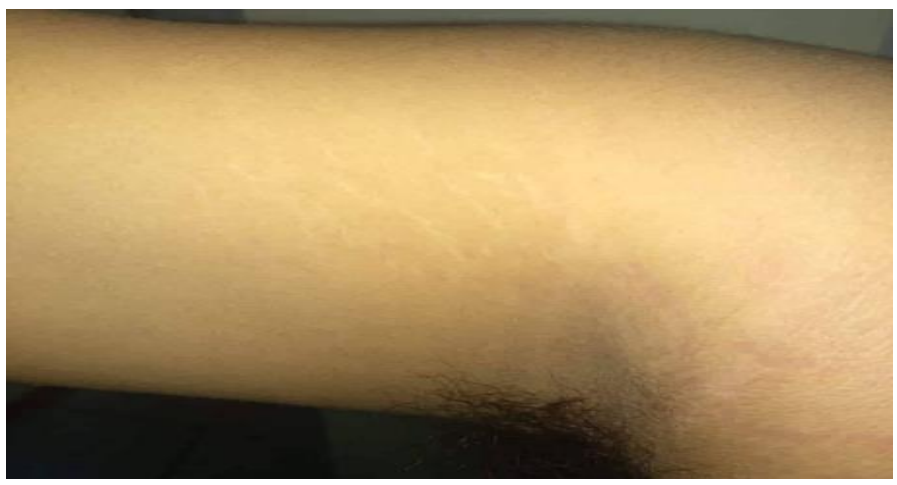

Fig. 13. Reduction Stretch marks in Axillary Nerve with the use of an Electro Stimulator.

\section{DISCUSSION}

The classic question of whether or not work the circuit, with the time developing a circuit be implemented that was tested physique. Hence the investigation split to make the paper established with the time one saw that if one could give this use that would reduce the level of stretch marks.

Other of the main drawbacks was the consumer of this product would be too, well thought as time. That using this would be excessive, which had to propose sessions a long time and use series as you see the improvement.

One thought about the disturbance of the aesthetic by which is also used as machine sport in theory if it is true. But the dimension of this paper is to demonstrate by testing that if one can improve and release of this congenital disease that occurs. Dare have too much time for rest or morbidly obese and thus could also see muscles dead, as dead skin by forming these whitish lines.

When the stimulation session is done, it should stimulate isometrically to thereby muscle is fixed and can block the movement that occurs muscle contraction spontaneously.

In the decade of the nineties it is discovered that with the percutaneous application of microloans current with certain pulses mounted to it, it is possible to generate a controlled local inflammatory response that undermines tissue repair, without systematic effect [8]. Currently, our electro stimulator allows a better result in less time through electromagnetic pulses.

Pixelated radiofrequency is based on the fractional unipolar radiofrequency emission to produce a double effect on both, the skin surface and depth [9]. We propose the use of the electro stimulator to produce muscle contractions in the skin.

To help prevent the appearance of stretch marks and varicose veins, and to be able to burn the unnecessary calories for her and the baby, and avoid the overweight of the pregnant woman [10]. Physical activity in pregnant women does not have to demand much physical wear to keep the baby's health in good condition.

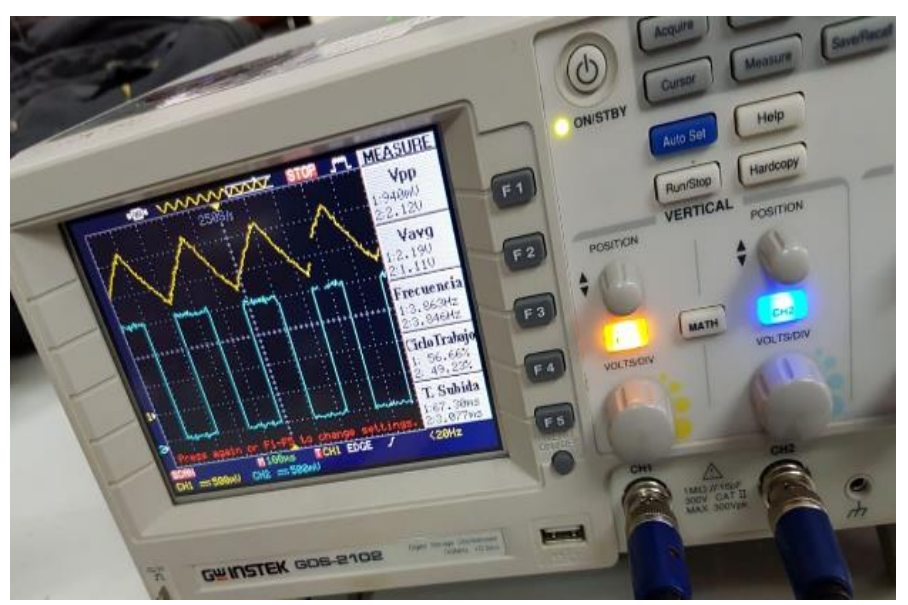

Fig. 14. Signal Square Wave and Triangular through Oscilloscope.

\section{CONCLUSIONS}

Is to explain everything about the definition, causes, and treatments of different types of stretch marks.

It was designed and implemented to a device of easy use to reduce stretch marks does not discriminate sex and age. The primary function of the electro stimulator is based on improving and toning the resilience of the skin.

It is essential to identify the degree of stretch marks level to give proper treatment with electrostimulation.

To have favorable outcomes with the use of electrostimulation treatment should be supplemented with functional exercises that can stimulate and accelerate the 
recovery of damaged skin. The idea is to have better muscle contraction as quickly as possible.

The frequency range for the correct use of electrostimulation was investigated to provide special treatment to the patient according to frequencies and stipulated.

The advantage of the electro stimulator is the reduction of stretch marks in the short term. It is also low cost and can be used by the population with small economic resources.

\section{REFERENCES}

[1] Teresa O. Rueda, "Securing non-ablative fractional laser $1540 \mathrm{~nm}$ " in Spain, May 2014 pp 25-26.

[2] Cruz A, "electrotherapy muscle strengthening" in Peru, pp 50-53, June 2018.

[3] Adalbert F. Armijos, "Design and implementation of a prototype of psychomotor early electrostimulation in children with the Down syndrome of the Despertasr Los Angeles center to improve motility in upper extremities" in Ecuador, pp. 20-21, October 2018.
[4] García, R. Marquez, S. Gomez, RI, \& Beltran, C, "Efficacy, safety, and cost-effectiveness of transcutaneous electrical nerve stimulation (TENS) for the treatment of skeletal muscle pain chronic" in Seville, pp. 5-7, April 2013.

[5] Pérez Fariñas L. "Treatment of Patient With abdominal obesity as prevention or progression of the metabolic syndrome." Cuban Journal of Physical Medicine and Rehabilitation. 2014; 6 (2-15).

[6] Leandro A. Cajina, Yanira Hernandez and Consuelo C. A. Rivera, "Application methods of electrotherapy in national hospitals and physical rehabilitation centers in the east, the Savior" in El Salvador, pp 41-42, October 2016.

[7] Martin R, "Form of the waves in low and medium frequency currents used in electrotherapy", pp. 35-63, Spain 2014.

[8] Mosquera T. Tatiana, " In vivo evaluation of the cosmetic efficacy of two biostimulation procedures with the application of platelet-rich plasma on stretch marks, to improve the elasticity and firmness of the treated skin" in Ecuador, pp 33-34, November 2017.

[9] Aguero Z. Fatima, Gonzalez B. Lourdes, "Treatment of stretch marks " in Paraguay, pp 86-88, August 2017.

[10] Anchundia M. Jean, "Benefit of physical activity to the pregnant woman from the perspective of nursing." in Ecuador, pp 8-11, August 2018. 